\title{
EDITORIAL
}

\section{The paradox in pneumococcal serotypes: highly invasive does not mean highly lethal}

\author{
M.W. Pletz*, T. Welte* and K.P. Klugman\#
}

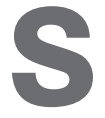

treptococcus pneumoniae is a major bacterial pathogen and a predominant cause of community-acquired pneumonia, acute exacerbations of chronic bronchitis, meningitis, sinusitis and bacteraemia in both developed and developing countries. Each year millions of people die from pneumococcal diseases. Even in developed countries, the casefatality rate of pneumococcal pneumonia is still high [1]. This is in contrast to the fact that 1) appropriate antibiotic treatment is available, and 2) to date, antimicrobial resistance rates of pneumococci for non-meningeal infections are still low in most countries or antimicrobial resistance (e.g. penicillin-resistance) is not related to treatment failure in pneumococcal pneumonia [2]. A considerable number of patients die within the first $48 \mathrm{~h}$ and these early deaths have not been prevented by antibiotic treatment [3].

BRUEGGEMANN et al. [4] investigated the risk of different pneumococcal serotypes to cause invasive disease. Invasive pneumococcal disease is defined as the isolation of $S$. pneumoniae from a normally sterile site, e.g. blood, cerebrospinal fluid or pleural fluid. The polysaccharide capsule protects pneumococci from phagocytosis and also may determine affinity to respiratory epithelium. Therefore, it seems reasonable that different pneumococcal serotypes exhibit different behaviour regarding colonisation and virulence. In that study, it was found that some serotypes have a much higher risk of causing invasive disease ("highly invasive") than others that are more frequently found as colonisers ("low invasive"). These results have been broadly confirmed by other groups (for review see [5]). In most studies, serotypes 6A, 6B, 19F and $23 \mathrm{~F}$ were identified as serotypes causing frequent nasopharyngeal colonisation but rarely invasive disease.

In several countries, large surveillance systems exist for invasive pneumococcal disease. Surveillance systems (e.g. USActive Bacterial Core Surveillance Study [6], German Pneumococcus Surveillance Study [7]) rely on invasive isolates, because here the causality between pathogen and disease is easy to establish. However, in pneumococcal pneumonia, a positive blood culture only can be obtained in a minority of patients $(\sim 10 \%)$ [8]. This means that the majority of pneumococci causing pneumonia are not detected by the previously

*Dept of Pulmonary Medicine, Hannover Medical School, Hannover, Germany. "The Rollins School of Public Health, Emory University, Atlanta, GA, USA.

CORRESPONDENCE: M.W. Pletz, Dept of Pulmonary Medicine, Hannover Medical School, Carl-Neuberg-Str. 1, 30625 Hannover, Germany. E-mail: pletz.mathias@mh-hannover.de mentioned surveillance systems. From a clinician's point of view, a positive blood culture or invasive disease is a marker for disease severity. Therefore, it seems appropriate to observe invasive pneumococcal isolates.

A future issue of the European Respiratory Journal (ERJ) will feature a study that suggests that less invasive strains may be more deadly [9]. The study investigates adult Spanish patients hospitalised with invasive pneumococcal pneumonia, and has identified infection by low invasive and not by highly invasive serotypes as an independent risk factor of mortality. The authors found that patients infected by highly invasive serotypes were predominately younger. However, using the age-independent American Thordcic Society (ATS) definition for severe pneumonia and controlling for age in a multiregression analysis, they confirmed infections by low invasive serotypes as an independent risk factor for mortality. The reason for this association was not uncovered in the study. However, the results are in line with another large study from Denmark. All of the serotypes associated with the high casefatality rate in adults were non-highly invasive serotypes [10]

A paper in this issue of $E R J$ reports a study from Mozambique dealing with pneumococcal pneumonia, which might, at least in part, explain the association between low invasive serotypes and a high case-fatality rate [11]. In a study in African children with invasive pneumococcal disease, the authors found an association between infection by low invasive serotypes and certain common genotypes with mannose-binding lectin (MBL) deficiency. MBL is one of the recognition modules of the lectin pathway of complement activation and plays a central role in the innate (i.e. early) immune response to pathogens. Some earlier studies failed to detect an association between MBL deficiency and invasive pneumococcal infections [12]. At first, the authors did not detect an association between pneumococcal infections and MBL deficiency; only after they had looked more specifically at the serotypes did they find such an association for low invasive serotypes. In contrast to the Spanish study, the authors regarded all serotypes not classified as highly invasive as low invasive. What does all this mean?

First, it is becoming clear that invasiveness is not equivalent to virulence (lethality). Pneumococci tend to infect patients with an impaired immune system: the very young with an immature immune system, the elderly who become immunosenescent and immunocompromised patients (e.g. HIV, post splenectomy, etc.) [13]. This "behaviour" of the pneumococcus seems to be even more pronounced for low invasive serotypes, 
which primarily infect older patients [9] or patients with MBL sufficiency [11].

The outcome of infectious diseases may be determined by both pathogen and host factors. The higher mortality of low invasive serotypes might, therefore, be determined by host rather than by pathogen-derived factors. The importance of host factors for the outcome of pneumococcal pneumonia has been underlined in several studies [1, 14].

Secondly, the Spanish study enrolled only patients with bacteraemic pneumonia. One could speculate that in nonbacteraemic pneumonia low invasive serotypes may lead even more frequently to high case-fatality rates. Thus, "bacteraemia", a necessary criterion for surveillance and serotyping, may mask significant pneumococcal infections associated with mortality due to low virulence strains causing non-bacteraemic pneumonia.

In conclusion, invasive infections in younger and nonimmunocompromised patients do not kill as often as less invasive strains infecting older and immunocompromised patients. Current surveillance strategies focusing on bacteraemia may not be sufficient to estimate the real burden of pneumococcal disease or the benefit of current pneumococcal vaccines in preventing pneumococcal mortality.

\section{STATEMENT OF INTEREST}

Statements of interest for all authors of this manuscript can be found at www.erj.ersjournals.com $/ \mathrm{misc} /$ statements.dtl

\section{REFERENCES}

1 Garcia-Vidal C, Ardanuy C, Tubau F, et al. Pneumococcal pneumonia presenting with septic shock: host- and pathogenrelated factors and outcomes. Thorax 2010; 65: 77-81.

2 Yu VL, Chiou CC, Feldman C, et al. An international prospective study of pneumococcal bacteremia: correlation with in vitro resistance, antibiotics administered, and clinical outcome. Clin Infect Dis 2003; 37: 230-237.
3 Austrian R, Gold J. Pneumococcal bacteremia with especial reference to bacteremic pneumococcal pneumonia. Ann Intern Med 1964; 60: 759-776.

4 Brueggemann AB, Griffiths DT, Meats E, et al. Clonal relationships between invasive and carriage Streptococcus pneumoniae and serotype- and clone-specific differences in invasive disease potential. J Infect Dis 2003; 187: 1424-1432.

5 Hausdorff WP, Feikin DR, Klugman KP. Epidemiological differences among pneumococcal serotypes. Lancet Infect Dis 2005; 5 : 83-93.

6 Nuorti JP, Butler JC, Farley MM, et al. Cigarette smoking and invasive pneumococcal disease. Active Bacterial Core Surveillance Team. N Engl J Med 2000; 342: 681-689.

7 Imohl M, Reinert RR, van der Linden M. Adult invasive pneumococcal disease between 2003 and 2006 in North-Rhine Westphalia, Germany: serotype distribution before recommendation for general pneumococcal conjugate vaccination for children $<2$ years of age. Clin Microbiol Infect 2009; 15: 1008-1012.

8 Chien YW, Klugman KP, Morens DM. Bacterial pathogens and death during the 1918 influenza pandemic. N Engl J Med 2009; 361: 2582-2583.

9 Luján M, Gallego M, Belmonte Y, et al. Influence of pneumococcal serotype group on outcome in adults with bacteremic pneumonia. Eur Respir J 2010; [Epub ahead of print DOI: 10.1183/09031936. 00176309].

10 Harboe ZB, Thomsen RW, Riis A, et al. Pneumococcal serotypes and mortality following invasive pneumococcal disease: a population-based cohort study. PLoS Med 2009; 6: e1000081.

11 Vallès X, Roca A, Lozano F, et al. Serotype-specific pneumococcal disease may be influenced by mannose-binding lectin deficiency. Eur Respir J 2010; 36: 856-863.

12 Kronborg G, Weis N, Madsen HO, et al. Variant mannose-binding lectin alleles are not associated with susceptibility to or outcome of invasive pneumococcal infection in randomly included patients. J Infect Dis 2002; 185: 1517-1520.

13 Steenhoff AP, Wood SM, Rutstein RM, et al. Invasive pneumococcal disease among human immunodeficiency virus-infected children, 1989-2006. Pediatr Infect Dis J 2008; 27: 886-891.

14 Alanee SR, McGee L, Jackson D, et al. Association of serotypes of Streptococcus pneumoniae with disease severity and outcome in adults: an international study. Clin Infect Dis 2007; 45: 46-51. 